\title{
Design, Modeling and Control of a Micromachined Nanopositioner with Integrated Electrothermal Actuation and Sensing
}

\author{
Y. Zhu, Member, IEEE, A. Bazaei, S. O. R. Moheimani, Fellow, IEEE, and M. R. Yuce, Senior \\ Member, IEEE
}

\begin{abstract}
In this paper, real-time feedback control of a novel micro-machined 1-degree-of-freedom (1-DoF) thermal nanopositioner with on-chip electrothermal position sensors is presented. The actuation works based on thermal expansion of silicon beams. The sensing mechanism works based on measuring the difference between the electrical resistances of two electrically biased identical silicon beams. The difference increases with displacement as the heat conductance of the sensor beams vary oppositely with position, resulting in different beam temperatures and resistances. The sensor pair is operated in differential mode to reduce low-frequency drift. The nanopositioner has a nonlinear static input-output characteristic. An open-loop controller is first designed and implemented. It is experimentally shown that uncertainties and sensor drift result in unacceptable nanopositioner performance. Hence, feedback control methods are necessary for accurate nanopositioning. A closed-loop feedback control system is designed using a proportional-integral (PI) controller together with the nonlinear compensator used for the open-loop control system. The closed-loop system provides acceptable and robust tracking performance for a wide range of set point values. For triangular reference tracking, which is needed in raster-scanned SPM, the tracking performance of the closed-loop system is further improved by incorporating a feed-forward controller.
\end{abstract}

Index Terms - Thermal actuation, thermal position sensing, microelectromechanical system (MEMS), nanopositioning, feedback control.

\section{INTRODUCTION}

$\mathrm{H}$ igh precision nanopositioners have been extensively used in many applications such as scanning tunneling microscopy (STM) [1], atomic force microscopy (AFM) [2], and emerging ultrahigh density probe storage system $[3,4]$. These nanopositioners typically have high positioning accuracy with a large dynamic range and a wide bandwidth, enabling fast and robust closed-loop position control. Although macro-scale

This research was funded by Australian Research Council (ARC) discovery grant- DP0774287.

Y.Zhu is with the School of Engineering, Griffith University, Australia (email: y.zhu@griffith.edu.au).

A.Bazaei, S.O.R.Moheimani, and M.R.Yuce are with the School of Electrical Engineering and Computer Science, the University of Newcastle, Australia (email: $\quad$ ali.bazaei, reza.moheimani, mehmet.yuce\}@newcastle.edu.au). nanopositioners can achieve nanometer-scale positioning resolution and accuracy, they are relatively large and expensive [5, 6]. Microelectromechanical System (MEMS) nanopositioners have attracted significant interest recently due to their small size, low cost, fast dynamics and the emergence of applications such as probe-based data storage [7, 8]. Closed-loop feedback control of the positioners is highly desirable if a high degree of displacement precision is required, and such a control system needs an accurate source of position information [9, 10]. However, many of the MEMS nanopositioners reported in the literature are not equipped with on-chip sensors due to the restrictions associated with micro-fabrication processes $[11,12]$. Thus, the in-plane movements are often measured by laser reflectance microscopes $[13,14]$ or optical microscopes [15], making the footprint of the whole system fairly large. There are several exceptions in the literature, e.g., an embedded on-chip capacitive displacement sensor was integrated in a thermally actuated positioner in [16]. Nevertheless, only open-loop results were obtained, and a complex fabrication process was required for electrical insulation between electrical heating and sensing circuits. Recently, a thermal sensing scheme was used in a probe-based storage device $[17,18]$. Micro-heaters were used to measure the motion of a MEMS micro-scanner with resolution of less than 1 $\mathrm{nm}$. Compared to a comb capacitive sensor, a thermal sensor is much more compact and can be easily integrated with actuators in a MEMS device [19]. In [20, 21], off-chip electromagnetic coil actuators were adopted for scanner actuation, and a complex mass-balanced structure was designed for vibration resistance purposes.

In this paper, a novel electrothermal position sensor is integrated with a electrothermal actuator in the same MEMS chip without the need for inclusion of extra electrical insulation fabrication process as reported in [16], or assembling two chips as reported in [20, 21]. Although compared with other MEMS actuators electrothermal actuators consume more power, they do offer certain advantages. They can operate under low voltages, can generate large forces, and enjoy a high degree of vibration resistance due to their stiff structures [22]. A MEMS device with integrated electrothermal actuation and sensing has been micro-fabricated in a bulk silicon process. Measurement results show that the positioner has a dynamic range of $14.4 \mu \mathrm{m}$ 
and an open-loop bandwidth of $101 \mathrm{~Hz}$. To reduce the low frequency thermal drift, the sensors are operated in a pair and measured by a differential circuitry. The sensor was measured to have a small low-frequency drift of $8.9 \mathrm{~nm}$ over 2000 seconds. The on-chip displacement sensing enables a feedback control capability. A model of the positioner is derived and a proportional-integral (PI) feedback controller is implemented digitally in a dSPACE rapid prototyping system to investigate the closed-loop performance of the positioner. Open-loop and closed-loop performance of the system in tracking a step displacement were investigated. The closed-loop step response results show a steady-state RMS positioning error of $8.6 \mathrm{~nm}$, while the corresponding value of the open-loop seek operation can reach to $1180 \mathrm{~nm}$. Good tracking performance was also achieved for a $10 \mathrm{~Hz}$ triangular reference by using a 2-DoF feedback control system consisting of a PI controller and a pre-filter.

The paper is organized as follows. In section II, we present the electrothermal positioning scheme and its modeling. Micro-fabrication process is described in this section as well. Section III presents the experimental setup and system characterization of the positioner. Open-loop static and dynamic responses are presented to verify that the positioner behaves as predicted. Section IV presents the details of the open-loop and closed-loop control design. The tracking performances of step and triangle references are presented in this section. Section V concludes this paper.

\section{Design, Modeling AND MicrofabricAtion}

\section{A. Design Description}

The conceptual schematic view of the nanopositioner is presented in Fig.1. The position sensors are two beam-shaped resistive heaters made from doped silicon. Application of a fixed dc voltage across the heaters results in a current passing through them, thereby heating the beams. As a heat sink, a rectangular plate is placed beside the beam heaters with a $2 \mu \mathrm{m}$ air gap. The positioner stage is actuated by an electro-thermal actuator.

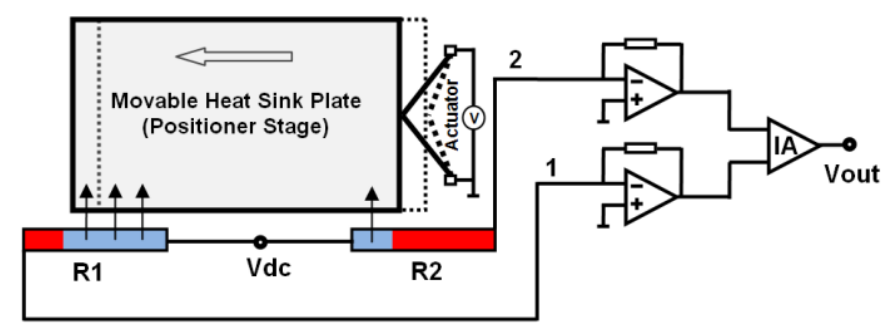

Fig. 1. Schematic diagram of the thermal position sensor with a differential amplifier circuit.

Before applying a voltage across the actuator, the positioner stage is at the initial rest position (dashed box in Fig.1), where the two edges of the sink plate are precisely aligned with the middle of the two thermal resistive sensors $R_{I}$ and $R_{2}$. The sensors are biased by a dc voltage source $V_{d c}$, and the heat generated in the resistive heater is conducted through the air to the heat sink plate (positioner stage). As the plate is centered between the two thermal sensors, the heat fluxes out of the sensors are identical, thereby equaling the temperature and resistance of the sensors. After applying a voltage on the actuator beams, the positioner stage is displaced towards left. The heat fluxes associated with the sensors on the left and on the right become different, resulting in a corresponding difference in the resistance of the left sensor $\left(R_{1}\right)$, and the right sensor $\left(R_{2}\right)$. Thus, the displacement information of the positioner stage can be detected by measuring the resistance difference between the two sensors. The differential changes of the resistance result in current variations in the beam resistors, and the currents are converted to an output voltage using a pair of trans-impedance amplifiers and an instrumentation amplifier. To suppress the common-mode noise, the gains of these two trans-impedance amplifiers must be well matched by adjusting the feedback resistance of the trans-impedance amplifiers. Employing the differential topology allows the sensor output to be immune from undesirable drift effects due to changes in ambient temperature or aging effects.

The positioner stage is actuated by a chevron electro-thermal actuator, as illustrated in Fig.1. Compared to other actuation methods, the electro-thermal actuators are simple to implement, operate at low voltage, and provide large forces. Due to the stiffness of the structures, no complex mass-balanced structures are needed for vibration resistance purposes, as proposed in [21]. The chevron electro-thermal actuator has a pair of thin hot arms at an angle with respect to each other as shown in Fig.1. The chevron actuator's operating voltage range is typically 0 to $15 \mathrm{~V}$, depending on the geometry. The displacement is proportional to $\mathrm{V}^{2}$, and the maximum displacement is limited by buckling of the hot arms at high temperatures $\left(>700^{\circ} \mathrm{C}\right)$ [14].

\section{B. Modeling}

In this section, we predict the sensor temperature and resistance as a function of sensor bias and actuator displacement. To simplify the analysis, lumped parameter approach and static (time-invariant) conditions are considered. Each sensor resistor is considered as a small solid object in which thermal convection is zero. This simplification allows us to describe the resistor temperature by a scalar variable rather than a distribution. Moreover, it allows us to combine different heat transfer coefficients between the object and different parts of its surrounding area (which includes air, heat sink, and gold pads at the ends) into an overall thermal resistance coefficient. According to Newton's law of cooling, the heat transfer from the object to air primarily occurs by thermal conduction in a very thin layer of air adjacent to the object surface rather than thermal convection. Although thermal convection can participate in the transfer of heat outside the foregoing thin layer, its overall affect in stationary conditions can be modeled as an equivalent thermal resistance between the object and air. Thus, we assume a uniform temperature distribution in each sensing resistor. The following discussion shows how lumped parameter analysis can predict the temperature in terms of the applied voltage. 


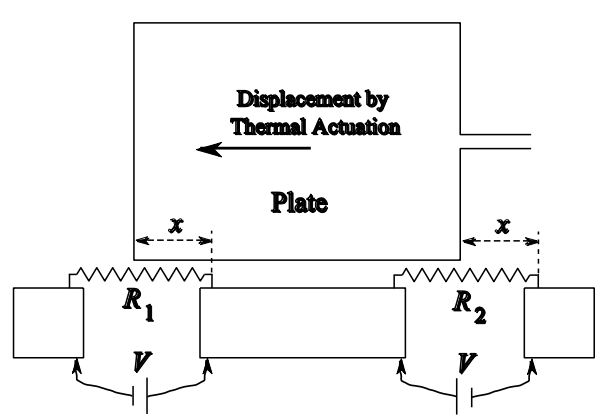

Fig. 2. Schematic diagram of the biased thermal sensors.

Let the electric resistance of a resistor at room temperature $T_{o}$ be $R_{o}(\Omega)$ and its variation with temperature described by a constant temperature coefficient $\alpha\left(1 /{ }^{\circ} \mathrm{C}\right)$. The overall thermal resistance between the resistor and the outside world, including its connection to a voltage source, is $K^{-1}\left({ }^{\circ} \mathrm{C} / \mathrm{W}\right)$. Assuming a uniform temperature distribution $T$ across the resistor, we can equate the electric power generated in the resistor with the thermal power flow from the resistor to the outside world as:

$$
\frac{V^{2}}{R_{o}\left[1+\alpha\left(T-T_{o}\right)\right]}=K\left(T-T_{o}\right)
$$

where $V$ is the constant voltage across the resistor. Since the constant $\alpha$ does not depend on temperature, Eq. (1) can be written as a second order algebraic equation with $T-T_{0}$ as an unknown. In this way, the resistance value and its temperature can be formulated in the following form:

$$
\begin{aligned}
& R=R_{o} \frac{1+\sqrt{1+\frac{4 \alpha V^{2}}{R_{o} K}}}{2} \\
& T=T_{o}+\frac{\sqrt{1+\frac{4 \alpha V^{2}}{R_{o} K}}-1}{2 \alpha}
\end{aligned}
$$

Referring to Fig.2, let us define $K_{1}^{-1}$ and $K_{2}^{-1}$ as the overall thermal resistance from electric resistors $R_{1}$ and $R_{2}$ of the sensor to the area around the sensor, respectively. As the plate in Fig.2 moves to the left side, $K_{I}$ increases while $K_{2}$ decreases. Let us assume a simple dependency between the overall thermal resistance and the plate position $x$, defined in Fig.2, in the following forms:

$$
\begin{aligned}
& K_{1}(\tilde{x})=K_{\text {min }}+\left(K_{\text {max }}-K_{\text {min }}\right) \tilde{x} \\
& K_{2}(\tilde{x})=K_{\text {min }}+\left(K_{\text {max }}-K_{\text {min }}\right)(1-\tilde{x})
\end{aligned}
$$

where $\tilde{x} \equiv x / L$ is the normalized position, $L$ is the length of each resistor, and $K_{\min }{ }^{-1}$ and $K_{\max }{ }^{-1}$ refer to minimum and maximum overall thermal resistance of each resistor. In this way, Equations (2) and (3) can be used to predict the steady-state values of resistors and their temperatures in the following form:

$$
\begin{aligned}
& R_{i}(\tilde{x}, V)=R_{o} \frac{1+\sqrt{1+\frac{4 \alpha V^{2}}{R_{o} K_{i}(\tilde{x})}}}{2}, \\
& T_{i}(\tilde{x}, V)=T_{o}+\frac{\sqrt{1+\frac{4 \alpha V^{2}}{R_{o} K_{i}(\tilde{x})}}-1}{2 \alpha}, i \in\{1,2\}
\end{aligned}
$$

where $R_{o}$ is each resistance value at zero bias voltage. At zero actuation, where $\tilde{x}=0.5$ and $K_{1}=K_{2}=\left(K_{\max }+K_{\text {min }}\right) / 2$, we assume that the sensor resistors match. Hence, the sensor output voltage is proportional to $R_{1}^{-1}-R_{2}^{-1}$. We employ this difference between electric conductances to measure the displacement as illustrated in the following simulation.

Assuming the parameter values in Table I and using Equations (4) and (5), the steady-state values of temperatures, resistances, and sensor output for different position values are obtained as shown in Fig. 3(a)-(c), respectively. Although the resistance values depend nonlinearly on displacement, the sensor output exhibits an almost linear dependency on displacement.

TABLE I.

Parameter values for Simulation

\begin{tabular}{ll}
\hline Parameter & Value \\
\hline$\alpha\left(\mathrm{K}^{-1}\right)$ & $3.7822 \times 10^{-4}$ \\
$R_{o}(\Omega)$ & 390 \\
$T_{o}\left({ }^{\circ} \mathrm{C}\right)$ & 27 \\
$K_{\min }(\mathrm{W} / \mathrm{K})$ & $8 \times 10^{-5}$ \\
$K_{\max }(\mathrm{W} / \mathrm{K})$ & $16 \times 10^{-5}$ \\
$V(\mathrm{~V})$ & 6 \\
\hline
\end{tabular}
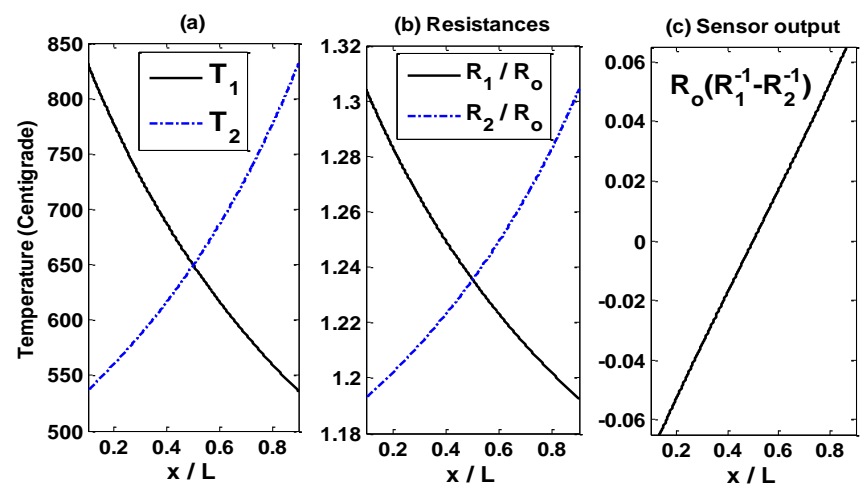

Fig.3. Steady-state values versus displacement for (a) resistor temperatures, (b) resistor values, and (c) normalized sensor output.

\section{Microfabrication}

The device was fabricated in a commercial Silicon-On-Insulator (SOI) MEMS foundry (MEMSCAP) process [23] with a $25 \mu \mathrm{m}$ thick silicon device layer and minimum gap of $2 \mu \mathrm{m}$. 


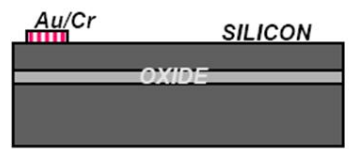

1) Metallization

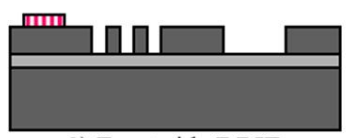

2) Front side DRIE

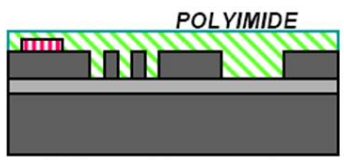

3) Front side protection

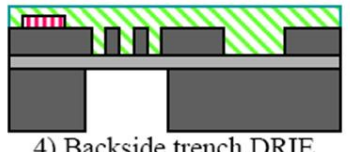

4) Backside trench DRIE

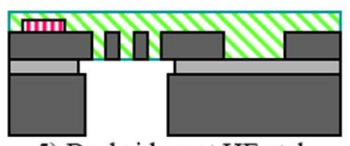

5) Backside wet HF etch

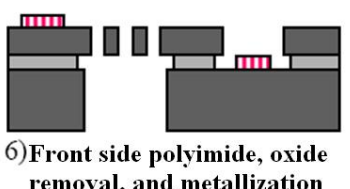

Fig.4. SOI MEMS fabrication processes from MEMSCAP

Fig. 4 provides an illustrated summary of this process:

1) Surface metal pads are patterned on a highly doped n-type $25 \mu \mathrm{m}$ silicon device layer to allow for ohmic contact.

2) Deep reactive ion etch (DRIE) from the front side of the wafer to define both the anchored and movable features of the structure.

3) A protective polyimide layer is applied to the front side.

4) A deep trench underneath the movable structures is created by etching through the substrate using DRIE.

5) The exposed buried oxide is removed using a wet HF etch.

6) The polyimide coat on the front side is removed by oxygen plasma, thereby allowing the movable structure to be fully released. The front side oxide layer is removed using a vapor HF process. Then a large contact metal pad (i.e. electrical grounded contact) is patterned on the substrate to reduce parasitics.

The Scanning Electron Microscope (SEM) image of the whole device and a section of it are provided in Fig.5.

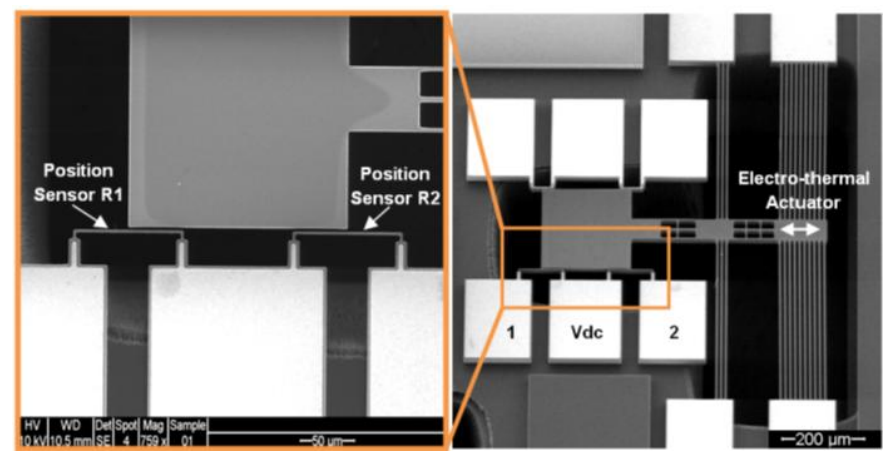

Fig.5. SEM images of the micromachined nanopositioner

To reduce the thermal coupling effects from the electro-thermal actuators to thermal sensors, a number of holes were embedded in the centre shuttle between actuators and the heat sink plate. The holes are expected to improve the thermal convection, thereby thermally insulating the heat sink plate from actuators. The suspension beams help the thermal insulation as well, due to the heat transfers to the substrate through the suspension beams and its anchors. Table II summarizes the device design parameters and measured beam resistance.

TABLE II.

Geometric ANd Material Properties

\begin{tabular}{cc}
\hline Parameter & Value \\
\hline Actuator beam width $(\mu \mathrm{m})$ & 5 \\
Actuator beam length $(\mu \mathrm{m})$ & $2 \times 400$ \\
Actuator beam angle $($ degree $)$ & 0.57 \\
Actuator beam quantity $($ pair) & 10 \\
Suspension beam width $(\mu \mathrm{m})$ & 5 \\
Suspension beam length $(\mu \mathrm{m})$ & 400 \\
Suspension beam angle $($ degree $)$ & 0 \\
Suspension beam quantity $($ pair $)$ & 3 \\
Sensor beam width $(\mu \mathrm{m})$ & 2 \\
Sensor beam length $(\mu \mathrm{m})$ & 100 \\
The gap between sensor and heat sink plate $(\mu \mathrm{m})$ & $200 \times 200$ \\
Device thickness $(\mu \mathrm{m})$ & 25 \\
temperature $(\Omega)$ & 388.9 \\
Measured resistance of thermal sensor R1 at room & 391.6 \\
temperature $(\Omega)$ & 116 \\
Measured resistance of thermal sensor R2 at room & \\
temperature $(\Omega)$ &
\end{tabular}

\section{EXPERIMENTAL CHARACTERIZATION}

The nanopositioner was calibrated using a Polytec ${ }^{\mathrm{TM}}$ Planar Motion Analyzer (PMA-400, Polytec GmbH). Digital image capturing and analysis methods were used to determine the in-plane displacement of the positioner stage. Although PMA uses a normal speed camera with a maximum exposure time of $82 \mathrm{~ms}$, it can measure periodic processes at frequencies as high as $1 \mathrm{MHz}$ by using the stroboscopic principle [24]. The step time response of the actuator was obtained using PMA, as illustrated in Fig.6. A $9 \mathrm{~V}$ step voltage was applied to the actuator, and the PMA recorded the displacement time response. After $20 \mathrm{~ms}$ of actuation, the positioner stage reached a stable position of $14.4 \mu \mathrm{m}$. The time constant is around $1.6 \mathrm{~ms}$.

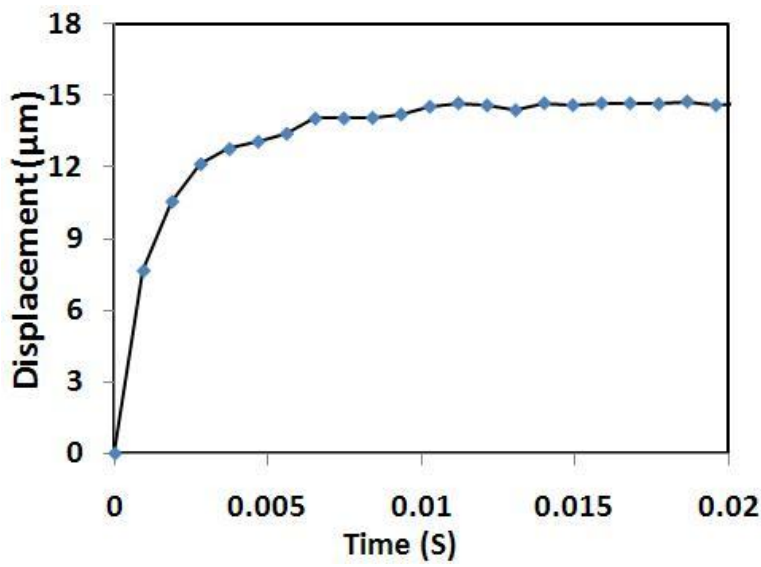

Fig.6. Displacement step time response of the actuator at an applied actuation voltage of $9 \mathrm{~V}$. 

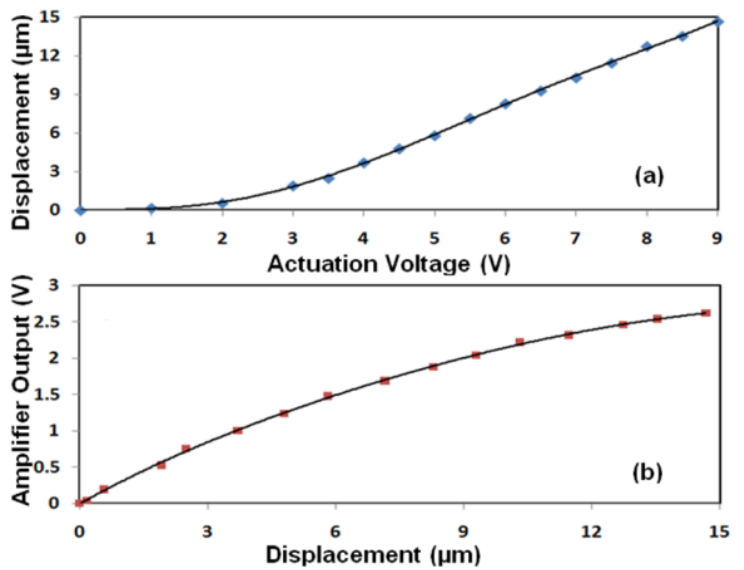

Fig.7. Experimental calibration results: (a) Actuator displacement versus actuation voltage. (b) Instrumentation Amplifier output versus actuator displacement.

Fig. 7 (a) shows the static actuation voltage vs. displacement measured data along with a fitted fifth order polynomial. With the actuation voltage of $9 \mathrm{~V}$, the electro-thermal actuator can achieve a maximum displacement of $14.4 \mu \mathrm{m}$. Due to the nature of the thermal expansion, the actuator can only move forward in one direction after application of a voltage. However, the actuator can be biased to an initial position by a de voltage, such that it can move forward or backward by increasing or decreasing the applied voltage. For instance, for a $10 \mu \mathrm{m}$ travel range with bi-directional movement, the actuator can be actuated by a $4.5-\mathrm{V}$ dc voltage to the middle position $(5 \mu \mathrm{m})$ of the desired range.

At every actuation voltage, the instrumentation amplifier outputs were recorded for calibration of the position sensors, as illustrated in Fig.7 (b). The sensors were biased with $6 \mathrm{~V}$, and the instrumentation amplifier gain was set at $90.3 \mathrm{~V} / \mathrm{V}$. At this bias voltage, each sensor has a power consumption of $60 \mathrm{~mW}$, and the sensitivity is $0.27 \mathrm{mV} / \mathrm{nm}$ at a displacement range of about $6 \mu \mathrm{m}$. The sensitivity decreases when the displacement is more than $6 \mu \mathrm{m}$. A possible reason is that the temperature distribution is not uniform in the thermal sensor beams. Due to the heat loss in the anchor of the sensor beams, the middle of the beam is hotter than its two ends, as noted in [25]. The temperature distribution can be seen in Fig. 8 . A $10 \mathrm{~V}$ dc voltage was applied to the left sensor, and illumination was observed in the middle of the silicon beam. The right sensor is dark since no voltage was applied to it.

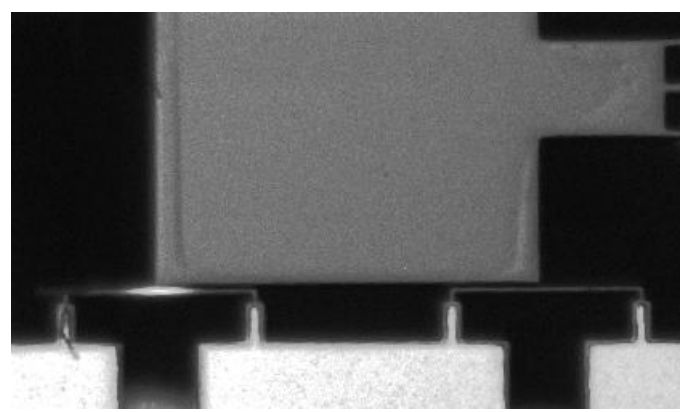

Fig.8. Optical micrograph of the position sensor's illumination at an applied voltage of $10 \mathrm{~V}$.

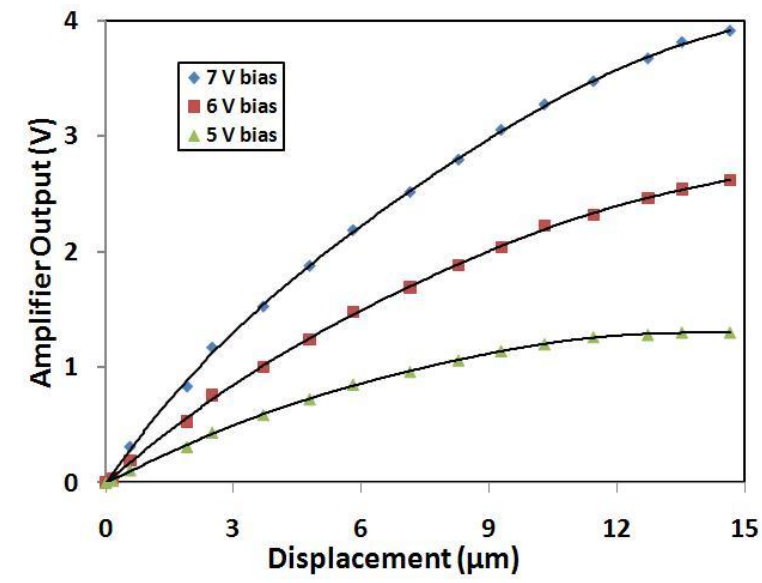

Fig.9. Amplifier output versus displacement at different sensor bias voltages of 5,6 , and $7 \mathrm{~V}$.

Fig. 9 illustrates the relationships between positioner displacement and sensor output at various sensor biases. The sensitivity can be increased by increasing the sensor bias voltage. However, the nonlinear nature of the response could not be eliminated by increasing the bias voltage.
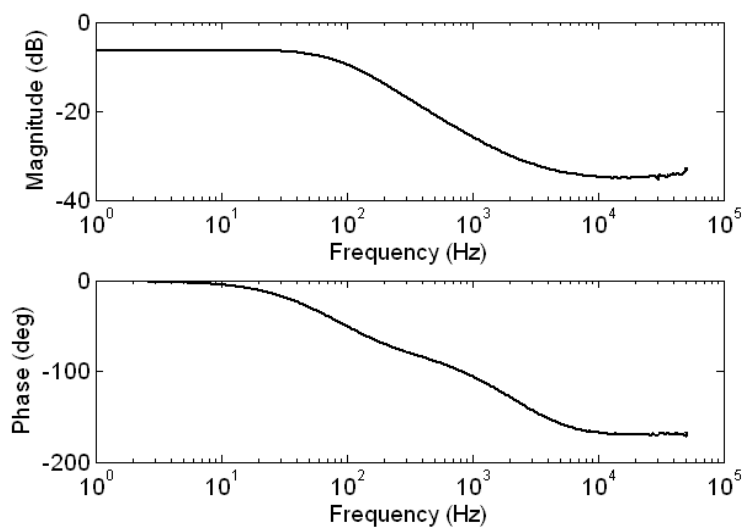

Fig.10. MEMS nanopositioner experimental frequency response.

The dynamic characterization was conducted using a HP35670A spectrum analyzer. A voltage of $4.5 \mathrm{~V}$ dc plus $1 \mathrm{~V}$ ac was applied to the actuator and swept sinusoidal measurements were obtained from $1 \mathrm{~Hz}$ to $51.2 \mathrm{kHz}$ using the on-chip thermal sensors. The frequency response, illustrated in Fig.10, shows that the open-loop bandwidth of the positioner is $101 \mathrm{~Hz}$, which is relatively low compared to the thermal structures fabricated by PolyMUMPs [26]. The reason is due to the fact that the heat transfer to surrounding air in SOIMUMPs is much slower than that to the substrate in PolyMUMPs. However, the bandwidth of $101 \mathrm{~Hz}$ is satisfactory for some positioning applications. 


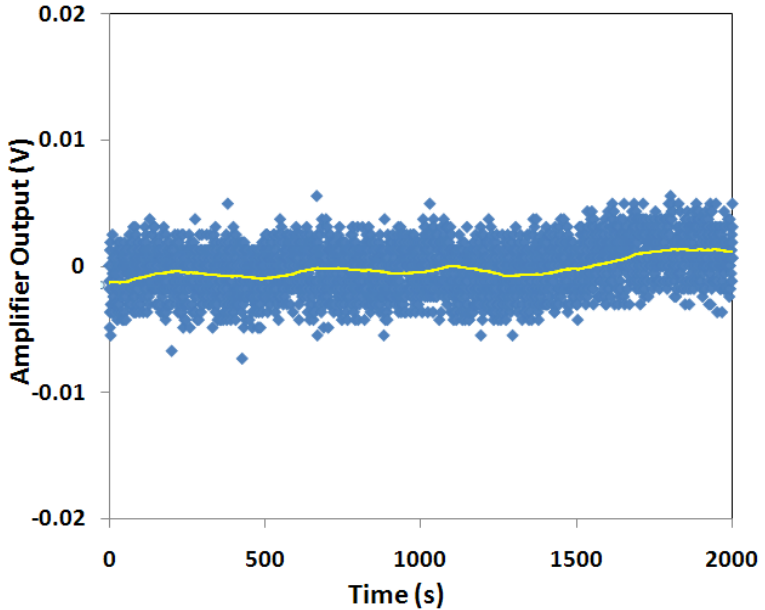

Fig.11. Experimental results of sensor drift (open-loop).

Fig. 11 shows the open-loop sensor drift when the positioner is at rest, which was measured at the output of the instrumentation amplifier over a period of 2000 seconds under normal laboratory conditions. The yellow/grey line is the moving average of the measured data, which indicates the low frequency drift. Thanks to the differential sensing of the sensor pair, the open-loop amplifier output has a low drift of $2.4 \mathrm{mV}$ over 2000 seconds, which corresponds to $8.9 \mathrm{~nm}$ displacement.

\section{CONTROL Design}

In this section, open-loop and closed-loop position control strategies are investigated for the positioner. A dSPACE-1103 rapid prototyping system was used for real time implementation of the controllers and data acquisition.

\section{A. Open-loop control}

Fig. 12 shows the structure of the open-loop control method. Look-up-tables can be used for fast computation of static nonlinear input-output mappings. For a specific input value to the block, it generates an output value based on interpolation or extrapolation among the recorded data values. The look-up-table at the input of the electro-thermal actuator converts the reference signal (in micrometers) to an appropriate actuation voltage, as shown in Fig. 13(a). Since the number and precision of measured data points are not enough to provide a smooth curve, a fifth order polynomial was fitted to the measured data in Fig. 7(a) to generate the data for the input look-up-table. A similar procedure was followed for the look-up-table at the output of the electro-thermal actuator to convert the sensor output voltage to displacement, as shown in Fig 13(b). For a sensor bias voltage of 7 volts, a third order polynomial was fitted to the measured data points in Fig. 9 to generate smooth and monotonic data for the output look-up-table. To avoid the infinite slope of the curve around zero displacement in Fig. 13(a), the origin for displacement was shifted to +1 micrometer. The performance of the open-loop control method with a stair-case reference signal is shown in Fig. 14. The open-loop controller cannot provide an acceptable tracking performance for displacement. This is due to plant uncertainties and sensor drift. Plant herein refers to the combination of the thermal MEMS device plus electronic circuits for actuation and sensing. Hence, feedback control is necessary for accurate positioning.

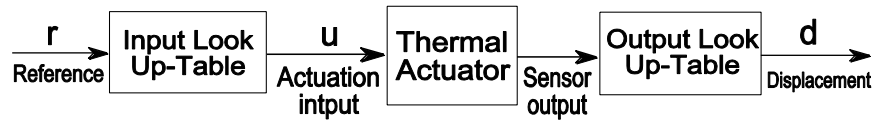

Fig.12. Block diagram of the open-loop control system.
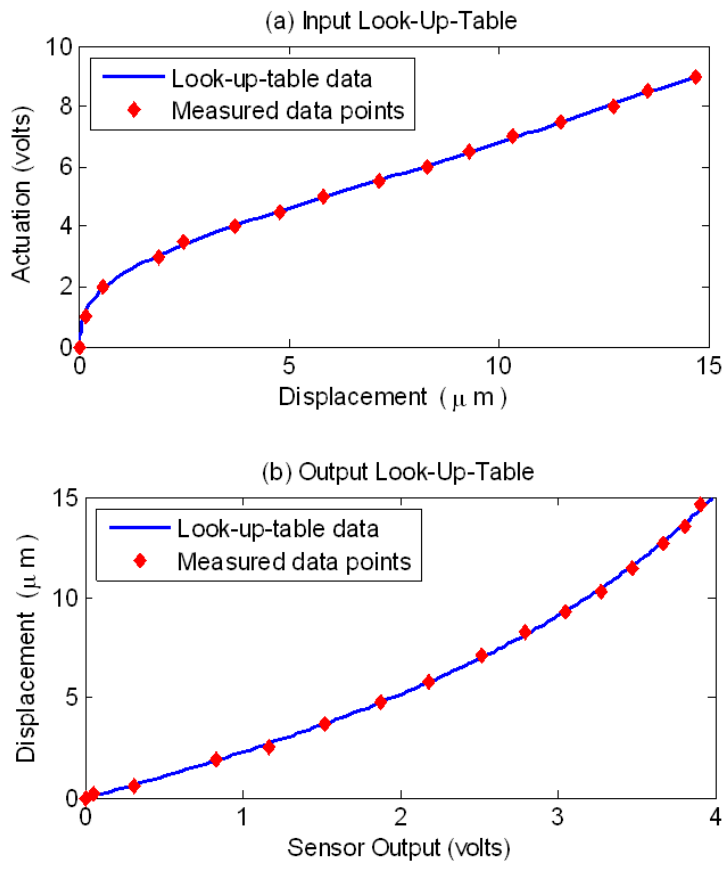

Fig.13. The data used in input and output look-up-tables.

(a)

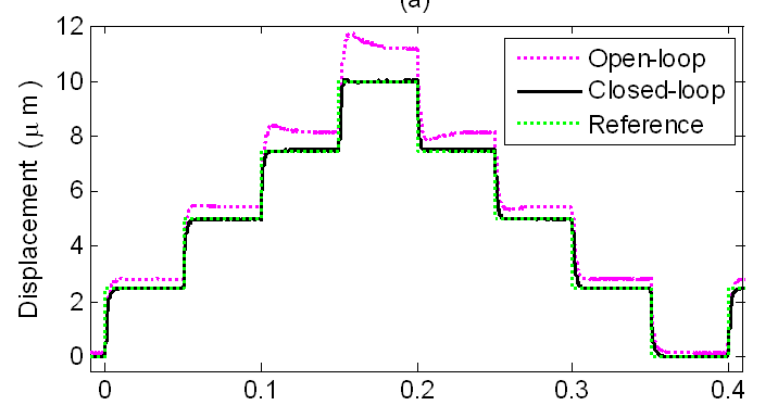

(b)

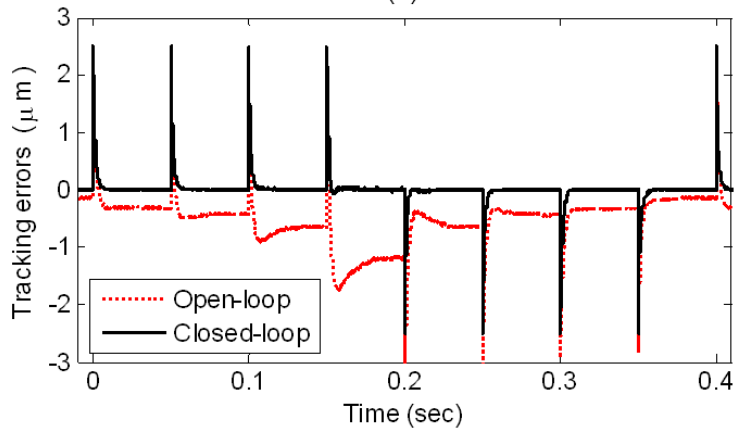

Fig.14. Tracking performances of open-loop and 1DoF closed-loop control methods for a stair case reference. 


\section{B. Closed-loop control.}

For closed-loop control, we incorporated a PI controller in addition to the nonlinear mappings, as illustrated in Fig. 15. The integration part in PI controller provides a closed-loop unity low frequency gain form reference to displacement for setpoint tracking and robustness to uncertainties and disturbances. The proportional part in the PI controller is used to reduce the overshoot in step response. With an integral gain of $k i=700$ and a proportional gain of $k p=1.3$, the positioning performance is significantly improved as shown in Fig. 14. Based on this control scheme, a controllable desired response of $2.5 \mu \mathrm{m}$ steps over a $10 \mu \mathrm{m}$ range was obtained with a maximum time constant of $1.4 \mathrm{~ms}$, as illustrated in Fig. 14. Fig. 16 is the close-up view of the $7.5 \mu \mathrm{m}$ closed-loop seek operation in Fig. 14, which indicates a steady-state RMS positioning error of $8.6 \mathrm{~nm}$. As a comparison, the steady-state RMS positioning error of a similar open-loop seek operation can reach to $1180 \mathrm{~nm}$.

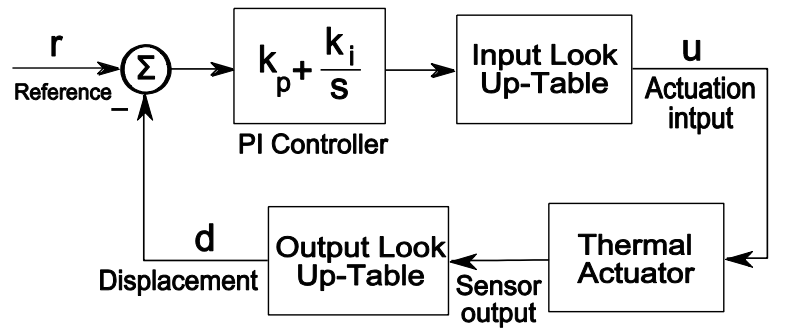

Fig.15. Block diagram of one-degree-of-freedom (1DoF) PI control system.

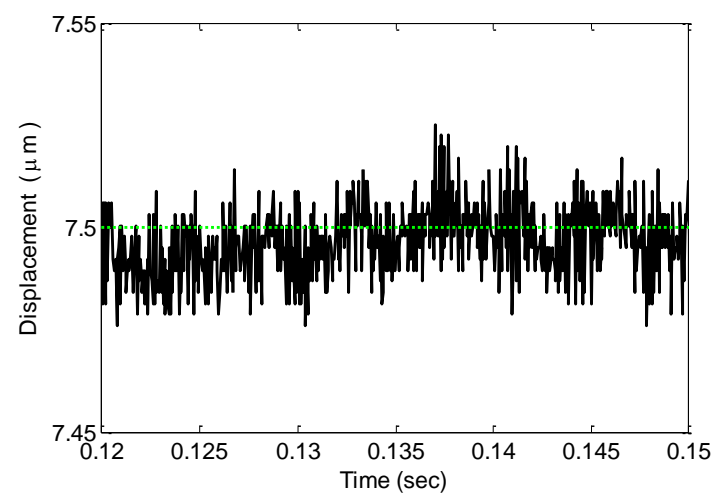

Fig.16. Close-up view of the $7.5 \mu \mathrm{m}$ closed-loop seek operation in Fig. 14.

For a $10 \mathrm{~Hz}$ triangular reference covering a $10 \mu \mathrm{m}$ range, the one-degree-of-freedom (1DoF) PI control system in Fig. 15, with $k_{i}=2000$ and $k_{p}=4$, yields an acceptable control performance, as shown in Fig. 17 (labeled "No prefilter"). In this case, the steady-state tracking error has a standard deviation of $0.09 \mu \mathrm{m}$, which is almost one percent of the $10 \mu \mathrm{m}$ full range. (a)

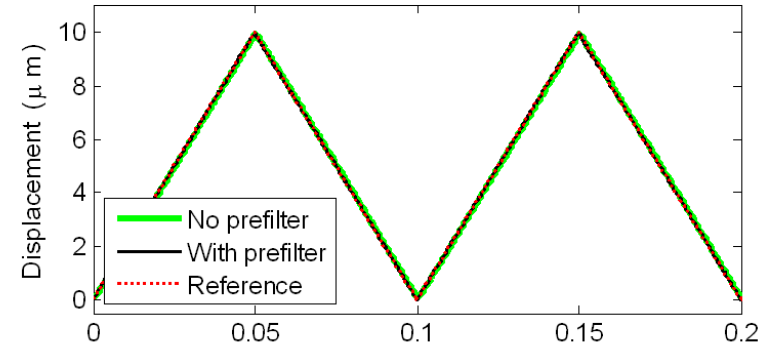

(b)

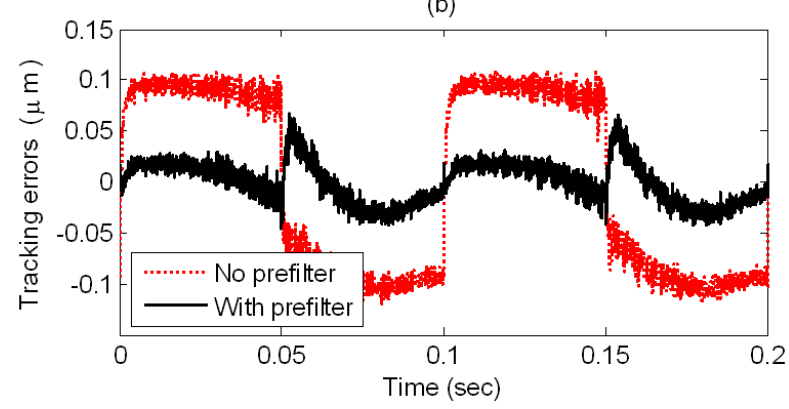

Fig.17. Performance of $1 \mathrm{DoF}$ (no prefilter) and $2 \mathrm{DoF}$ (with prefilter) control systems in tracking of a triangular reference.

The control performance can be further improved with a 2DoF control system consisting of a PI controller and a prefilter, as shown in Fig. 18. The look-up-tables provide the nonlinear mappings described earlier. To provide better stability margins we used lower gains of $k_{i}=1100$ and $k_{p}=2$ for the PI controller. This also reduces the effect of measurement noise on the controlled displacement output due to feedback. The prefilter with the frequency response shown in Fig. 19, is used to speed up the response and reduce the tracking error without affecting the stability margins and unity low-frequency gain of the closed-loop system. The tracking performance for a $10 \mathrm{~Hz}$ triangular reference is shown in Fig. 17 (labeled "With prefilter"). It can be observed that displacement output closely follows a desired triangular reference within a wide range of 10 $\mu \mathrm{m}$ with a standard deviation of $0.02 \mu \mathrm{m}$.

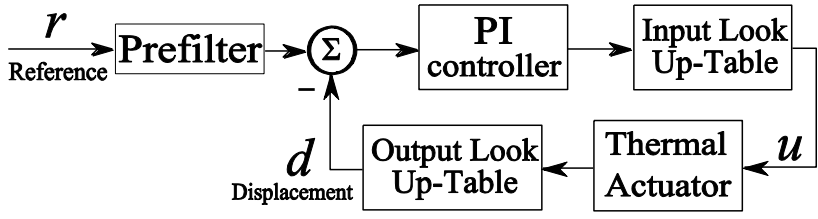

Fig.18. Two-degree-of-freedom (2DoF) feedback control structure. 

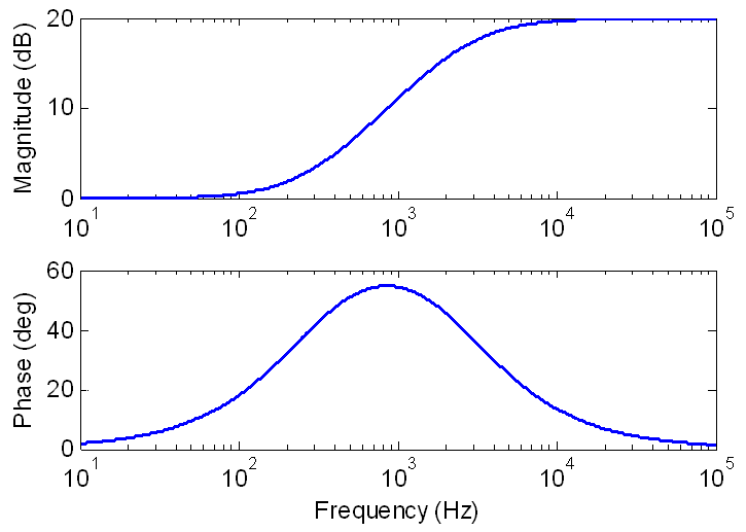

Fig.19. Bode diagram of the prefilter in the $2 \mathrm{DoF}$ control systems.

Results of sinusoidal reference tracking, with $1 \mathrm{DoF}$ and 2DoF control structures are also shown in Fig. 20 with labels "No prefilter" and "With prefilter", respectively, where the prefilter and look-up-tables described earlier were used again. The integral and proportional gains for the $1 \mathrm{DoF}$ control system were $k_{i}=4000$ and $k_{p}=20$, and for the $2 \mathrm{DoF}$ control system were $k_{i}=1250$ and $k_{p}=2.2$. In each case, the gain values were tuned to achieve the best tracking performance. Similar to the triangular reference case, the $2 \mathrm{DoF}$ control system provides less tracking error, less noise bandwidth, and more stability margins with respect to the $1 \mathrm{DoF}$ system. The steady-state positioning error for a full seek range of $10 \mu \mathrm{m}$ has standard deviations of 0.05 $\mu \mathrm{m}$ and $0.02 \mu \mathrm{m}$ with the $1 \mathrm{DoF}$ and $2 \mathrm{DoF}$ systems, respectively.

(a)

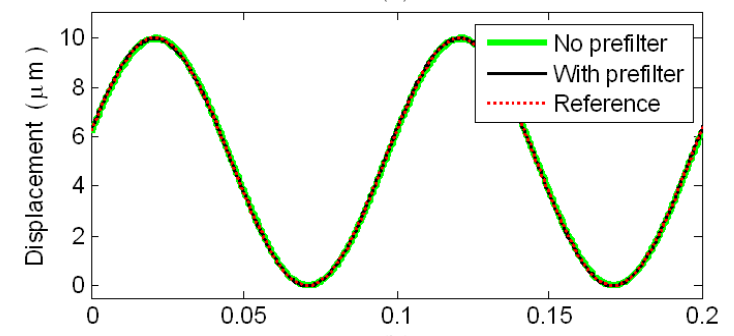

(b)

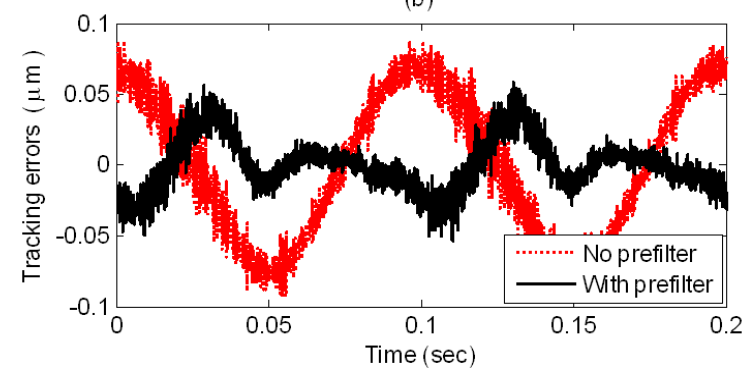

Fig.20. Performance of $1 \mathrm{DoF}$ (no prefilter) and $2 \mathrm{DoF}$ (with prefilter) control systems in tracking of a sinusoidal reference.

\section{CONCLUSION}

A novel micromachined silicon nanopositioner with on-chip electro-thermal actuator and sensor has been presented with nanometer resolution and low sensor drift. The MEMS positioner was embedded in a feedback loop to realize a precise position control. Due to the nonlinear nature of the electro-thermal actuator, a nonlinear inversion block was added to the feedback loop to linearize the plant. The experimental results showed that the positioner with the PI controller achieved a high degree of positioning accuracy with good robustness.

The actuator positions the heat-sink plate, which is a suitable platform for samples located on it as a load. This one-dimensional positioner is a preliminary experimental device for a future two-dimensional one, which will be used as a nano-positioner stage for scanning probe microscopy. As the forces induced by thermal expansion of the arms are strong, it is expected that the performance of the positioner would not significantly change if small probes or samples are attached to the platform. Due to the limitation of the SOIMUMPs fabrication process, it is not possible to design a two-dimensional thermal based positioner in a single device layer. However, the thermal sensors and actuators can be placed in different layers, which can be fabricated in METALMUMPs process through MEMSCAP. A 2DOF thermal based nanopositioner is under investigation by the authors for imagining applications.

\section{ACKNOWLEDGMENT}

The authors would like to thank David Phelan and Deming Zhu from the school of Medical Sciences at University of Newcastle for their help on SEM images.

\section{REFERENCES}

[1] G.Binning, and H.Rohrer, "The scanning tunneling microscope," Sci.Am., vol.253, pp.50-56, 1986.

[2] G.Binning, C.Quate, and C.Gerber, "Atomic force microscope," Phys. Rev. Lett., vol.56, no.9, pp.930-933, 1986.

[3] A.Pantazi, A.Sebastian, G.Cherubini, M.Lantz, H.Pozidis, H.Rothuizen, and E.Eleftheriou, "Control of MEMS-Based scanning-probe data-storage devices," IEEE Trans. on Nanotechnology, Vol.15, No.5, pp.824-841, 2007.

[4] A.Pantazi, M.A.Lantz, G.Cherubini, and H.Pozidis, and E.Eleftheriou, "A servomechanism for a micro-electro-mechanical-system-based scanning-probe data storage device," Nanotechonology, 15, pp.S612-S621, 2004

[5] Y.K.Yong, S.S.Aphale, and S.O.R.Moheimani, "Design, Identification, and Control of a Flexure-Based XY Stage for Fast Nanoscale Positioning," IEEE Trans. on Nanotechnology, Vol.8, No.1, pp.46-54, 2009.

[6] Y.K.Yong, S.S.Aphale, and S.O.R.Moheimani, "Atomic force microscopy with a 12-electrode piezoelectric tube scanner," Review of Scientific Instruments, Vol.81, No.3, pp033701, 2010.

[7] C.H.Kim, and Y.K.Kim, "Micro XY-stage using silicon on a glass substrate," J.Micromech. Microeng., Vol.12, pp.103-107, 2002.

[8] C.K.Pang, Y.Lu, J.Chen, H.Zhu, J.Yang, J.Mou, G.Guo, B.M.Chen, and T.H.Lee, "Design, fabrication, sensor fusion, and control of a micro $\mathrm{X}-\mathrm{Y}$ stage media platform for probe-based storage systems," Mechatronics, Vol.19, pp.1158-1168, 2009.

[9] S.Devasia, E.Eleftheriou, and S.O.R.Moheimani, "A survey of control issues in nanopositioning," IEEE Trans. on control systems technology, vol.15, no.5, pp.802-823, 2007.

[10] A.Sebastian, and D.Wiesmann, "Modeling and experimental identification of silicon microheater dynamics: a systems approach," J. of MEMS, Vol.17, No.4, pp.911-920, 2008.

[11] L.Gu, X.Li, H.Bao, B.Liu, Y.Wang, M.Liu, Z.Yang, and B.Cheng, "Single wafer processed nanopositioning XY-stage with trench-sidewall 
micromaching technology," J.Micromech.Microeng., Vol.16, pp.1349-1357, 2006

[12] C.S.B.Lee, S.Han and N.C.MacDonald, "Single crystal silicon (SCS) XY-stage fabricated by DRIE and IR alignment," Proceeding of IEEE MEMS2006, pp.28-33.

[13] J.J. Gorman, Y-S. Kim, and N.G. Dagalakis, "Control of MEMS nanopositioners with nano-scale resolution," Proceeding of IMECE2006, Chicago, IIlinois USA, November 5-10, 2006.

[14] R.Hichey, D.Sameoto, T.Hubbard, and M.Kujath, "Time and frequency response of two-arm micromachined thermal actuators", J.Micromech.Microeng., 13, pp.40-46, 2003.

[15] Y.Sun, M.A.Greminger, D.P.Potasek, and B.J.Nelson, "A Visually Servoed MEMS Manipulator," Experimental Robotics VIII, Springer Berlin/Heidelberg, pp.255-264, 2003.

[16] L.L. Chu, and Y.B. Gianchandani, "A micromachined 2D positioner with electrothermal actuation and sub-nanometer capacitive sensing", $J$. Micromech. Microeng., 13, pp.279-285, 2003.

[17] M.A.Lantz, G.K.Binning, M.Despont, and U.Drechsler, "A micromechanical thermal displacement sensor with nanometre resolution," Nanotechnology, 16, pp.1089-1094, 2005.

[18] G.K.Binning, M.Despont, M.A.Lantz, and P.Vettiger, "Thermal movement sensor," International patent application, WO 2004/020328 A1.

[19] N.B.Hubbard, M.L.Culpepper, and L.L.Howell, "Actuators for micropositioners and nanopositioners," Transactions of the ASME, Vol.59 November 2006, pp.324-334.

[20] A.Sebastian, A.Pantazi, S.O.R. Moheimani, H.Pozidis, and E.Eleftheriou, "Achieving subnanometer precision in a MEMS-based storage device during self-servo write process," IEEE Tran. on nanotechnology, vol.7, no.5, pp.586-595, 2008.

[21] M.A.Lantz, H.E.Rothuizen, U.Drechsler, W.Haberle, and M.Despont, "A vibration resistant nanopostioner for mobile parallel-probe stroage applications," $J$. microelectromechanical systems, vol.16, no1, pp.130-139, 2007.

[22] V.Kaajakari, Practical MEMS. Las Vegas, NV: Small Gear Publishing, 2009.

[23] http://www.memscap.com/en_mumps.html

[24] Software Manual, Planar Motion Analyzer Software 2.3, Polytec

[25] U.Durig, "Fundametals of micromechanical thermoelectric sensors," Journal of Applied Physics, 98,044906, 2005.

[26] C.D.Lott, T.W.McLain, J.N.Harb, and L.L.Howell, "Modeling the thermal behavior of a surface-micromachined linear-displacement thermomechanical microactuator," Sensors and Actuators A, 101, pp.239-250, 2002.

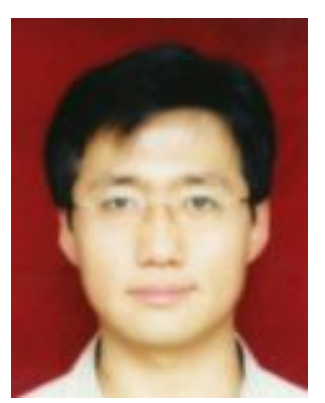

Yong Zhu received a Ph.D degree in Microelectronics from the Peking University, Beijing, China, in 2005. He worked as a Research Associate in Department of Engineering, University of Cambridge, UK, in 2006 and 2007. From 2008 to 2011, he was a Research Academic in the School of Electrical Engineering and Computer Science, the University of Newcastle, Australia. Currently he holds lecturer position in the School of Engineering, Griffith University, Australia.

His research interests include micromachined resonators, power harvesters, nanopositioners, capacitive sensors, RF MEMS, Mechatronics, as well as interface circuits design for MEMS.

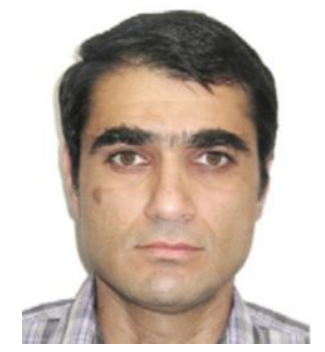

Ali Bazaei holds Ph.D. degrees from University of Western Ontario, London, ON, Canada, and from Tarbiat Modares University, Tehran, Iran, and M.Sc. and B.Sc. degrees from Shiraz University, Shiraz, Iran, all in Electrical Engineering, in Jan. 2009, Jun. 2004, Aug. 1995, and Feb. 1992, respectively. From Sep. 1995 to Jan. 2000, he was an instructor with Yazd University, Yazd, Iran. From Sep. 2004 to Dec. 2005, he was a Research Assistant at the Department of Electrical and Computer Engineering, University of Western Ontario, London, ON, Canada. He is currently a Research Academic with the School of Electrical Engineering and Computer Science, The University of Newcastle, Australia. His research interests lie in the general area of nonlinear systems including control and modeling of structurally flexible systems, friction modeling and compensation, and neural networks.

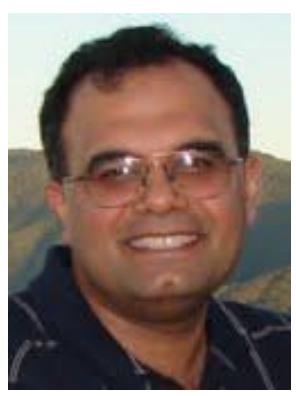

S. O. Reza Moheimani received a Ph.D. in electrical engineering from University of New South Wales at the Australian Defence Force Academy, Canberra, Australia in 1996. Since 1997 he has been with University of Newcastle, Australia, where he is currently a Professor and Australian Research Council Future Fellow in the School of Electrical Engineering and Computer Science. He has served on the editorial boards of a number of journals, including IEEE Transactions on Control Systems Technology, IEEE/ASME Transactions on Mechatronics and Control Engineering Practice. He is a recipient of the 2007 IEEE Transactions on Control Systems Technology Outstanding Paper Award and the 2009 IEEE Control Systems Technology Award. He is a Fellow of the Institute of Physics and a Fellow of IEEE. His current research interests include applications of control and estimation in nanoscale positioning systems for scanning probe microscopy, control of microactuators in MEMS and data storage systems.

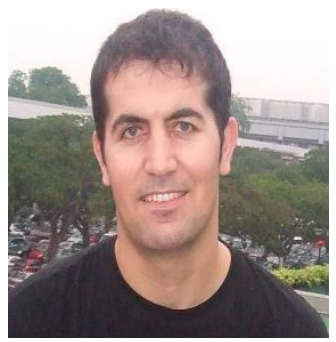

Mehmet Rasit Yuce (S'01-M'05-SM'09) received the M.S. degree in Electrical and Computer Engineering from the University of Florida, Gainesville, Florida in 2001, and the Ph.D. degree in Electrical and Computer Engineering from North Carolina State University (NCSU), Raleigh, NC in December 2004. Currently he holds senior lecturer position in the School of Electrical Engineering and Computer Science, University of Newcastle, New South Wales, Australia. 
Between August 2001-October 2004, he has served as a research assistant with the Department of Electrical and Computer Engineering at NCSU, Raleigh, NC. He was a post-doctoral researcher in the Electrical Engineering Department at the University of California at Santa Cruz in 2005. His research interests include wireless implantable telemetry,

Wireless Body Area Network (WBAN), bio-sensors, analog/digital mixed signal VLSI for wireless, biomedical, and RF applications. Dr. Yuce has published about 70 technical articles and received a NASA group achievement award in 2007 for developing an SOI transceiver. He is a senior member of IEEE. 